\title{
Focusing the beam of a compact, repetitively pulsed $x$-ray laser to study the interaction of radiation with metallic targets and $x$-ray reflectometry
}

\author{
I A Artyukov, B R Benware, A V Vinogradov, Yu S Kas'yanov, \\ V V Kondratenko, C D Macchietto, A Ozols, J J Rocca, J L A Chilla
}

\begin{abstract}
The interaction of focused soft $x$-ray laser radiation with metallic targets was studied experimentally. The radiation of a capillary-discharge Ne-like Ar ion laser $(\lambda=46.9 \mathrm{~nm})$ with a repetition rate of $1 \mathrm{~Hz}$ was focused on the surface of metallic samples by using a spherical $\mathrm{Si}$ / Sc multilayer mirror. The radiation intensity at the target surface was far greater than the threshold for laser ablation of the materials in use - aluminium, brass, and stainless steel. A study of the shape of laser craters in relation to the position of the caustic of the focusing mirror, combined with computer ray-tracing simulations, made it possible to determine the radiation intensity distribution in the focal region of the mirror. The radiation energy density in the $2 \mu \mathrm{m}$ central domain of the focal spot was $10^{2} \mathrm{~J} \mathrm{~cm}^{-2}$, and the corresponding intensity was $10^{11} \mathrm{~W} \mathrm{~cm}^{-2}$. The reflectivity of a Si/Sc multilayer mirror was measured as a function of the angle of incidence of the radiation.
\end{abstract}

\section{Introduction}

The specific nature of lasers generating soft $x$ rays (SXR) (the absence of a resonator and the inhomogeneity of the amplifying medium) calls for a thorough study of the space-time and energy characteristics of their radiation, its degree of coherence, spectral composition, etc. In this work, we studied the spatial intensity distribution of the laser beam in the caustic of a focusing spherical mirror from the shape of craters produced in the targets irradiated in the laser facility described in Ref. [1]. We also considered applications of the SXR laser in reflectometry.

\section{Laser ablation by focusing SXR radiation}

Appreciable radiation intensities can be attained by focusing SXR beams, opening up new opportunities for applications.

I A Artyukov, A V Vinogradov P N Lebedev Physics Institute, Russian Academy of Sciences, Leninskiĩ prospekt 53, 117924 Moscow, Russia; B R Benware, C D Macchietto, A Ozols, J J Rocea, J L A Chilla Colorado State University, Fort Collins, CO 80523, USA;

Yu S Kas'yanov Institute of General Physics, Russian Academy of Sciences, ul. Vavilova 38, 117942 Moscow, Russia;

V V Kondratenko Kharkov State Polytechnical University, ul. Frunze 21, 310002 Khar'kov, Ukraine

Received 29 October 1999

Kvantovaya Elektronika 30 (4) 328 - 332 (2000)

Translated by E N Ragozin; edited by M N Sapozhnikov
In a recent experiment [2], a nanojoule pulse of a recombination $\mathrm{Li}$-like $\mathrm{Al}$ ion laser $(\lambda=15.47 \mathrm{~nm})$ was focused down to a submicron-sized spot with an intensity of $\sim 10^{7} \mathrm{~W} \mathrm{~cm}^{-2}$. Preliminary results on the focusing of high-energy SXR laser pulses were reported in Ref. [3].

In this section we are concerned with studying the radiation intensity distribution in the focal region of a focused laser beam with a wavelength of $46.9 \mathrm{~nm}$ generated by a capillary-discharge Ne-like Ar ion laser. The results of the first experiments on the ablation of various materials by SXR laser radiation are also given here. The experimental arrangement to study the radiation intensity distribution in the focal region is shown schematically in Fig. 1. The laser pulses were generated in the Ar plasma of an $18.2 \mathrm{~cm}$ long capillary discharge produced by short current pulses. The parameters of the capillary discharge are given elsewhere [1]. The laser pulse energy was about $0.13 \mathrm{~mJ}$, and the duration was $1.2 \mathrm{~ns}$. The far-field pattern of the laser radiation was annular in shape, with an angular divergence $\sim 4.6 \mathrm{mrad}$ between the maxima [1]. The annular beam shape was due to the radiation refracted by the radial density gradients in the amplifying medium $[4,5]$. The laser beam was focused by a spherical multilayer mirror with a radius of curvature $R=10 \mathrm{~cm}$ placed $256.5 \mathrm{~cm}$ from the output aperture of the capillary in the vacuum chamber.

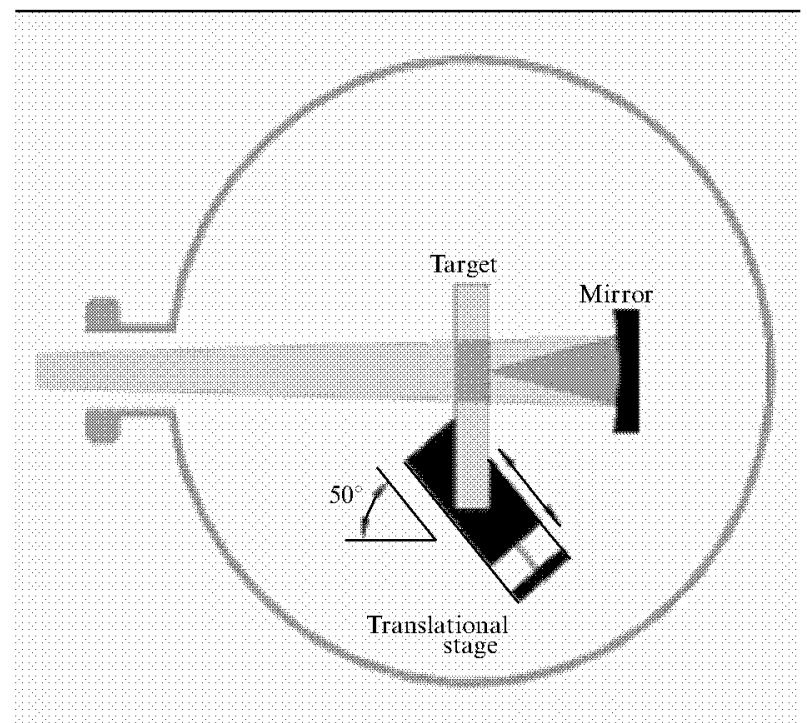

Figure 1. Schematic of the experimental setup for studying the ablation and the radiation distribution under SXR focusing. 
The multilayer mirror coating was deposited by magnetron sputtering on a ultra-smooth borosilicate substrate with a diameter of $2.5 \mathrm{~cm}$ and a roughness height $\sim 0.1 \mathrm{~nm} \mathrm{[6].} \mathrm{The}$ mirror reflectivity at a wavelength of $46.9 \mathrm{~nm}$ was $43 \%$ for normal incidence of radiation (see Section 2). In order to minimise off-axis aberrations, the mirror plane was aligned normal to the incident beam. The reflected beam was focused on the edge of a thin $(1.6 \mathrm{~mm})$ metallic plate, which served as a target. Given this irradiation scheme, the target intercepted a part of the beam incident on the mirror. However, this did not introduce significant changes because the beam diameter was about $12 \mathrm{~mm}$ and only a small portion of the beam was rejected.

The intensity of the focused laser beam was sufficiently high to cause the ablation of aluminium, brass, and stainless steel in the paraxial domain within a distance of several hundred micrometres from the focus. The characteristics of a crater on a metallic surface depend not only on the radiation distribution, but also on the pulse length, the melting temperature, and the heat conductivity of the metal [7]. Nevertheless they make it possible to characterise the two-dimensional intensity distribution in the focused laser beam in sufficient detail. The intensity distribution along the optical axis was determined by translating the target-bearing stage at an angle to the axis by using a computer-controlled step motor.

Sequences of laser beam imprints on the target surface were obtained for different positions of the target relative to the optical beam axis. Each sequence was accomplished for a continuous motion of the translational stage and a laser pulse repetition rate of $1 \mathrm{~Hz}$. Fig. 2 (top) shows the photograph of ablation imprints on the surface of a brass target obtained with the aid of a scanning electron microscope. It embraces the range of convergence of the laser beam, beginning with distances $\sim 450 \mu \mathrm{m}$ to the focus.

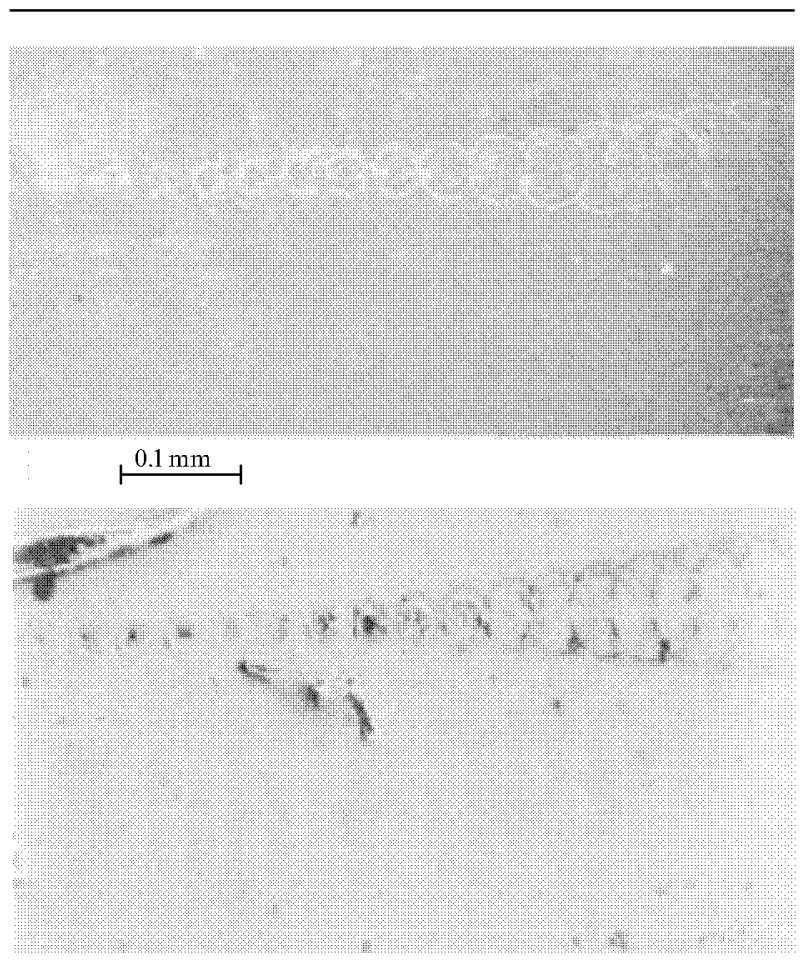

Figure 2. Ablation imprints on the brass (top) and steel (bottom) targets. Each imprint resulted from exposure to a single laser pulse.
Fig. 2 shows that the material ablation increases as the target is shifted from the mirror to the focus. Two neighbouring spots are spaced at $22.2 \mu \mathrm{m}$ along the optical axis. Note that this spacing is far less than the displacement due to the sample motion perpendicular to the optical axis. Each
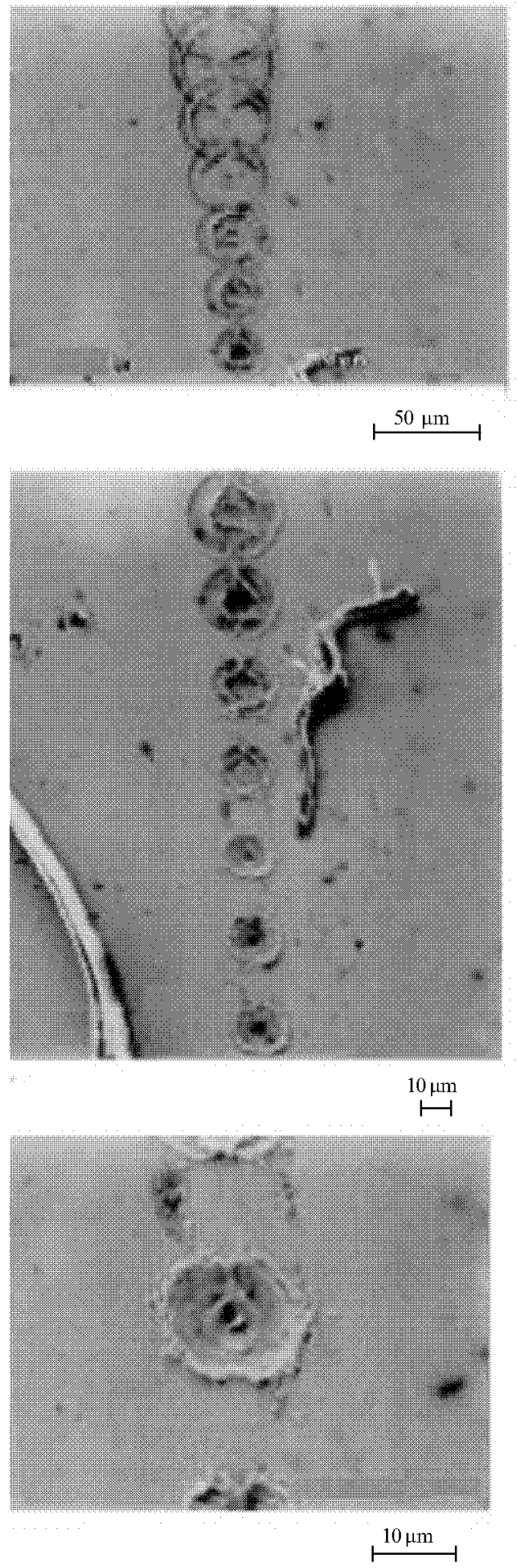

Figure 3. Ablation imprints on the brass target as it was moved from the mirror to the focus. Each imprint was produced by a single pulse of laser irradiation. 
imprint results from the action of a single laser pulse. The sequence of ablation imprints on the surface of a stainless steel sample is shown in Fig. 2 (bottom).

Fig. 3 shows a photograph of the surface of a brass target at high magnification. At a distance of several hundred micrometres from the focus, the ablation imprints have the shape of narrow rings of circular symmetry, disrupted near the region where the beam incident on the mirror was intercepted by the target. As the focal region is approached, the dimension of the ablation rings increases, and a central spot appears. The depth of ablation rings measured with a ZYGO profilometer was about $2 \mu \mathrm{m}$. The ablation imprint near the focus degenerates into a typical laser crater. The external diameter of the smallest spot was equal to approximately $17 \mu \mathrm{m}$ and the diameter of the indentation in it was $\sim 2-3 \mu \mathrm{m}$. The ablation imprints in the domain located behind the focus, which are not shown in Fig. 3, soon become blurred and hardly discernible.

We obtained the quantitative characteristics of the radiation distribution in the focal region by the computeraided method of ray tracing. A point source located $263 \mathrm{~cm}$ from the mirror was treated as the radiation source. Its angular radiation distribution was specified in such a way as to describe both the far-field and the near-field distributions of laser radiation known from the experiment [4]. Note that the same result was obtained when we used the model of a finite source $(300 \mu \mathrm{m})$. The paths of 13000 rays were traced to simulate the beam propagation. The radial distribution of radiation intensity in the focal region thus obtained is shown in Fig. 4; the black dots mark the boundaries of the ablation domain determined from the experiment.

One can see from Fig. 4 that the ray-tracing technique provides an adequate description of all the most significant features of the profile of ablation imprints. Our calculations showed that the high ray density in the focal region measuring several hundred micrometres determines the thin annular domains where the main part of the beam energy is concentrated. As the focus is approached, a central peak begins to dominate in the energy distribution, which is also in complete agreement with the experiment. The similar radiation distribution pattern is typical of the spherical aberrations.

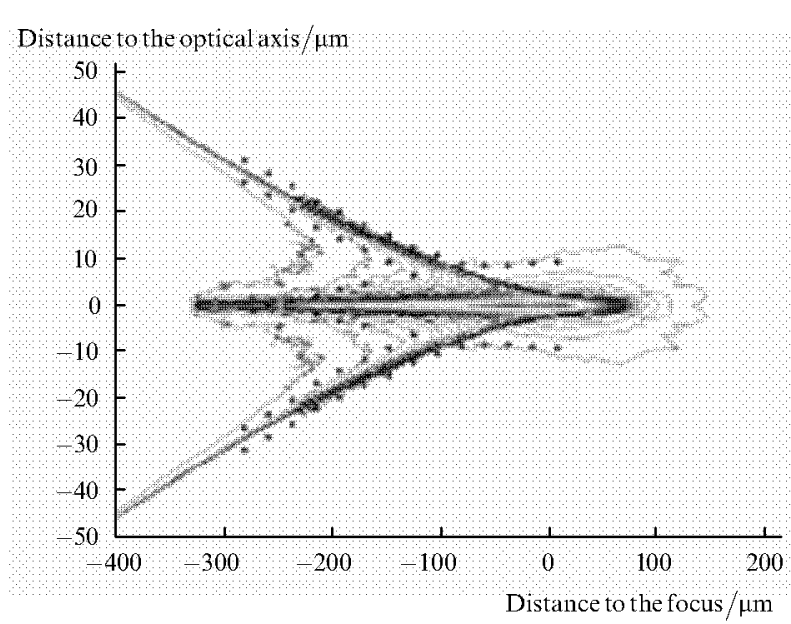

Figure 4. Isolines of the intensity distribution of laser radiation in the focal region of the mirror obtained by numerical ray tracing. The dots indicate the boundaries of the ablation region determined experimentally.
Note that the beam structure observed in the focal region is not necessarily a consequence of the annular shape of the initial beam. When the most remote rays begin to converge to the optical axis, the radiation distribution is dominated by the sharp central peak, which corresponds to the indentation seen in the photographs. The average radiation intensity in this domain $2 \mu \mathrm{m}$ in diameter, calculated from the fraction of rays that intersect this domain, was evaluated as $10^{11} \mathrm{~W} \mathrm{~cm}^{-2}$. Our estimates show that the minimum size of the focal spot obtained in the experiment is determined by the spherical aberration of the focusing mirror [8].

A schematic of the experimental setup for studying the ablation upon repeated irradiation of a target is shown in Fig. 5. The laser beam is reflected from a plane $\mathrm{Si} / \mathrm{Sc}$ multilayer mirror which has an opening $3 \mathrm{~mm}$ in diameter at its centre. Through this opening, the radiation is focused onto the target with the aid of a spherical $\mathrm{Si} / \mathrm{Sc}$ multilayer mirror with a radius of curvature $R=20 \mathrm{~cm}$. Although the beam intensity is reduced after reflection from the plane mirror with a reflectivity of $\sim 40 \%$, its intensity at the target proves to be above the ablation threshold within a spot several hundred micrometres in diameter. Repeated target irradiation results in the formation of deep craters due to ablation. Shown at the left of Fig. 6 is a sequence of laser craters on an aluminium target, which were obtained by exposure to 40 pulses at each target position. At the right of Fig. 6, the image of a hole approximately $10 \mu \mathrm{m}$ in diameter obtained near the focus is shown.

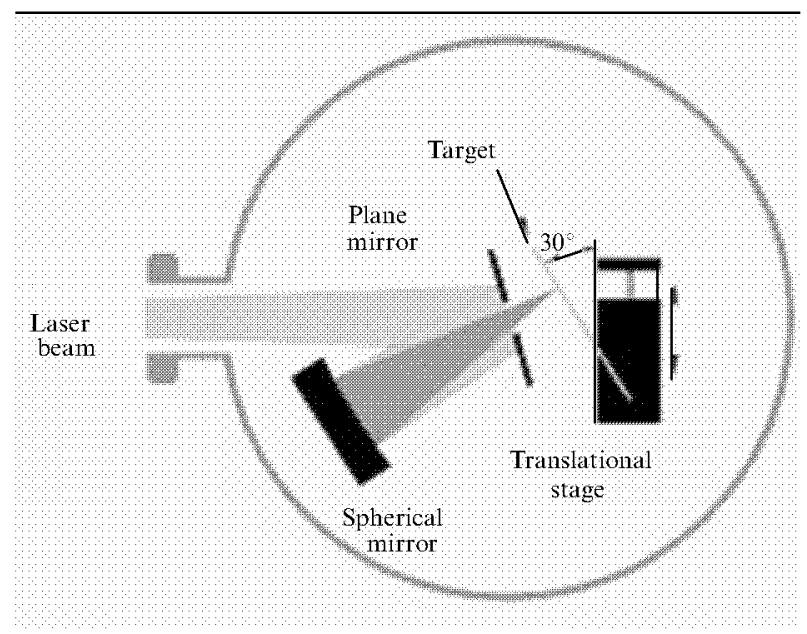

Figure 5. Schematic of the experimental setup for studying the ablation produced by repeated target irradiation by laser pulses.

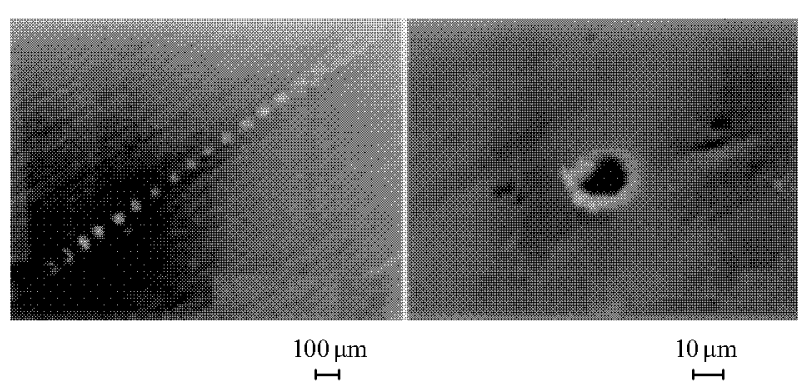

Figure 6. Ablation imprints on an aluminium target (on the left) as it moves from the mirror to the focus. The hole on the right of Fig. 6 was produced by exposure to 40 laser pulses in the vicinity of the focus. 


\section{SXR reflectometry}

The experimental setup for measuring the reflectivities of materials is shown in Fig. 7. The radiation source was a capillary-discharge laser oscillating at a wavelength of $46.9 \mathrm{~nm}$. The experiment was performed in a vacuum chamber, at a distance of $150 \mathrm{~cm}$ from the exit aperture of the capillary. The samples were mounted on the axis of a bench stage rotated with a step motor, the angle of rotation being varied from zero to $90^{\circ}$. The intensity of the reflected beam was detected with a vacuum photodiode (1) mounted on a movable arm, which followed the angular motion of the reflected beam. To limit the dimensions of the laser beam incident on a sample, an aperture stop of diameter $1 \mathrm{~mm}$ was placed at the entrance of the chamber, which is of significance for small grazing angles.

To carry out absolute reflectivity measurements, the intensity of the reflected beam was normalised to the intensity of the incident beam for every laser shot. Toward this end, a reference beam was formed by means of a beam-splitter, which was made of a gold-plated mesh with a transmittance of $50 \%$. The mesh transmittance was measured with an error of less than $0.5 \%$. The intensity of the reference beam was measured by a vacuum photodiode ( 2 ). The angular dependence of reflectivity was determined by scanning the angle of incidence upon irradiation of the sample by laser pulses with a repetition rate of $1 \mathrm{~Hz}$. The signals from the photodiodes
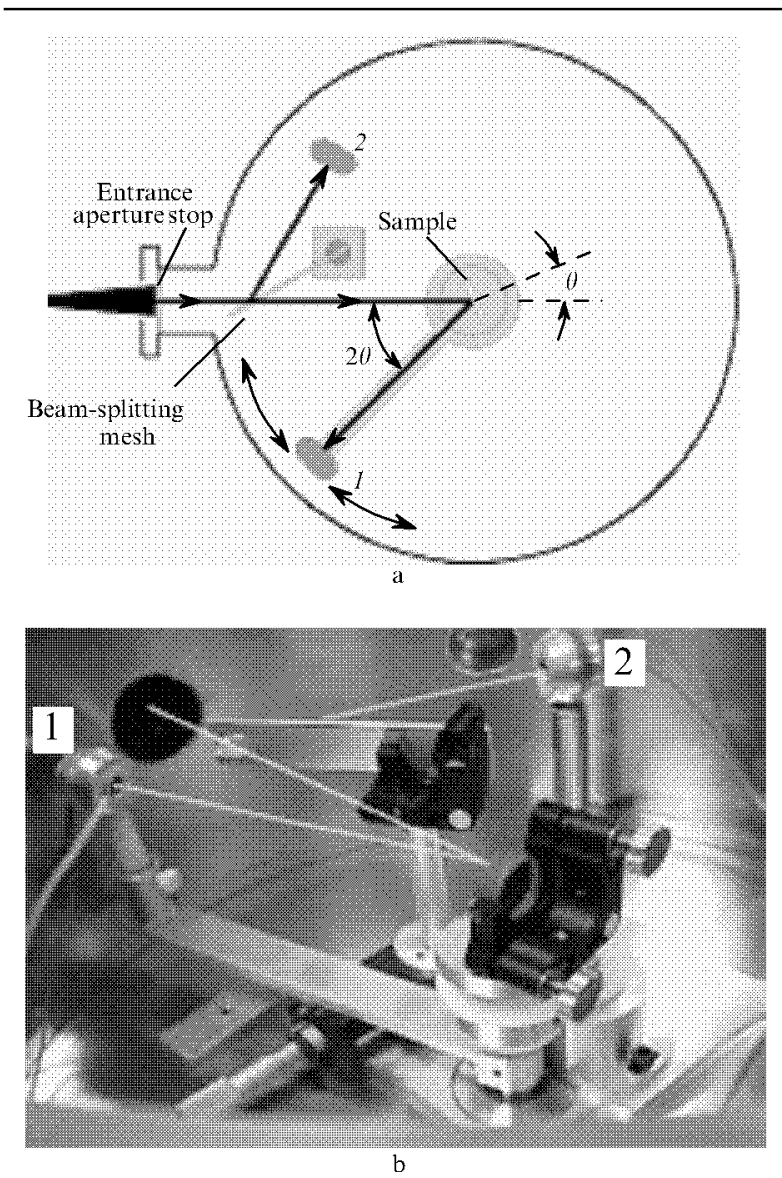

Figure 7. Schematic diagram (a) and photograph (b) of the setup for laser reflectometry on the basis of a low-bulk, repetitively pulsed x-ray laser. were recorded and stored with a digital oscilloscope (HewlettPackard Model 54825A).

Fig. 8 illustrates the dependence of the reflectivity on the angle of incidence for polished crystalline silicon. Prior to being placed into the vacuum chamber, the sample was processed for $5 \mathrm{~min}$ in a $5 \%$ aqueous solution of $\mathrm{HF}$ and then washed in acetone and methanol. The curve was obtained in 300 laser shots, which is typical of the experiments conducted. For near-normal angles of incidence, the photodiode screened a sample from the incident beam which limited the minimum measurable angle at a level of $1.6^{\circ}$. This angle corresponds to the first experimental point near the normal and is determined by the setup geometry; all subsequent angular readings were made relative to this angle. In another limiting case, when the angles of incidence approach $90^{\circ}$, the projection of the beam incident on a sample becomes greater than the sample dimension. This limits the maximum angle at which the experimental data can be obtained. The $1 \mathrm{~mm}$ diameter of the incident beam restricts the maximum angle of incidence to $85.5^{\circ}$.

The same setup was used to determine the angular dependence of the reflectivity of a $\mathrm{Si} / \mathrm{Sc}$ multilayer mirror at the wavelength $\lambda=46.9 \mathrm{~nm}$. A multilayer coating with a period $18-27 \mathrm{~nm}$ and the ratio of layer thicknesses $H(\mathrm{Sc}) / H(\mathrm{Si})=0.786$ was deposited by magnetron sputtering on a borosilicate substrate. The parameters of such a mirror were earlier discussed in detail in Ref. [6]. Fig. 9 shows the angular dependence of the reflectivity for a mirror designed and fabricated to operate at wavelength $\lambda=46.9 \mathrm{~nm}$ at normal incidence. The plot is an average of four series of measure-

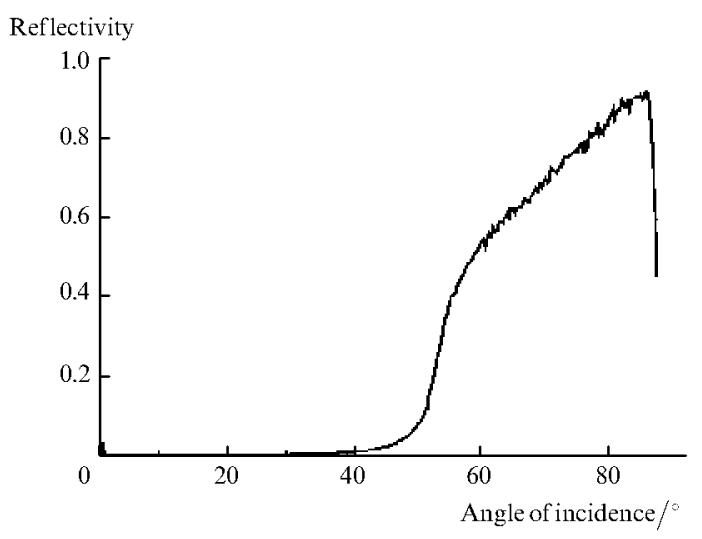

Figure 8. Reflectivity of crystalline $\mathrm{Si}$ as a function of the angle of incidence.

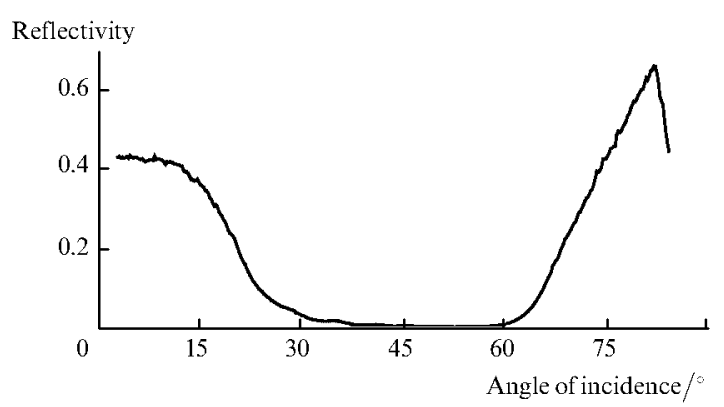

Figure 9. Reflectivity of a Si/Sc multilayer mirror at the wavelength $\lambda=46.9 \mathrm{~nm}$ as a function of the angle of incidence. 
ments. These series differed in the number of readings, which ranged from 200 to 400 when the angle of incidence was varied from 0 to $90^{\circ}$. Each series of measurements yielded similar results, and the averaging was performed merely to reduce random noise. Note that the reflectivity for the angle of incidence of $1.6^{\circ}$ was equal to $43 \%$. The sharp decrease in the reflectivity at angles above $80^{\circ}$ results from the fact that the beam projection on the mirror becomes greater than the beam size. We emphasise that the use of a repetitively pulsed laser in reflectivity measurements simplifies the procedure and reduces the measurement time significantly.

\section{Conclusions}

We performed for the first time experiments on the ablation of different materials by focused SXR laser radiation. A spherical multilayer mirror was employed to focus the laser radiation. For a pulse energy of $0.13 \mathrm{~mJ}$, the radiation intensity in the focal spot was limited primarily by the spherical aberration and was as great as $10^{11} \mathrm{~W} \mathrm{~cm}^{-2}$. Owing to the high repetition rate of laser pulses, the procedure of reflectivity measurements in the SXR range was made considerably simple. Measurements were made of the reflectivity of a $\mathrm{Si} / \mathrm{Sc}$ multilayer mirror as a function of the angle of radiation incidence.

Acknowledgements. The authors thank Yu A Uspenskii and $\mathrm{Yu} \mathrm{P}$ Pershin for helpful discussions. This work was supported by the National Science Foundation (NSF Grant No. DMR-9512282). The authors also acknowledge the support of the Civilian Research and Development Foundation of the USA (CRDF Grant No. RP1-240), which made it possible to develop, through international cooperation, the multilayer optics for our experiments. The participants in this work at Colorado State University express their gratitude to the Colorado Institute of Modern Technologies.

\section{References}

1. Benware B R, Macchietto C D, Moreno C H, Rocca J J Phys. Rev. Lett. 815804 (1998)

2. Ohchi T, Yamaguchi N, Fujikawa C, Hara T Proceedings of the Sixth International Conference on X-Ray Lasers (Bristol: IOP Publishing, 1998) p. 683

3. Zeitoun Ph, Sebban S, Murai K, Tang H, et al. Proceedings of the Sixth International Conference on X-Ray Lasers (Bristol: IOP Publishing, 1998) p. 677

4. Moreno C H, Marconi M C, Shlyaptsev V N, Benware B R, Macchietto C D, Chilla J L A, Rocca J J, Osterheld A L Phys. Rev. A 581509 (1998)

5. Chilla J L A, Rocca J J J. Opt. Soc. Am. B: Opt. Phys. 132841 (1996)

6. Uspenskiı Yu A, Levashov V E, Vinogradov A V, Fedorenko A I, Kondratenko V V, Pershin Yu P, Zubarev E N, Fedotov V Yu Opt. Lett. 23771 (1998)

7. Miller J C, Haglund R F Laser Ablation and Desorption (San Diego: Academic Press, 1998)

8. Marconi M C, Chilla J L A, Moreno C H, Benware B R, Rocca J J. Phys. Rev. Lett. 792799 (1997) 